\title{
A Review Analysis of Insomnia: Age, Gender, Career, and Efficient Behavior Methods Perspectives
}

\author{
Perugu Shyam¹, Momina S Mohammad²
}

\begin{abstract}
Insomnia is one of the sleep disorders. Most of the people neglect the symptoms of insomnia. Our aim is to analyze an overall all together at one-step review of defining insomnia, types of insomnia, symptoms of insomnia, other diseases relating to insomnia, factors, diagnosis, and treatments of insomnia is performed for the scope of innovative method for solving the problem. Literatures were reviewed to analyze the insomnia statistically in terms of gender, age, and career. It is observed that women at their postmenopause state are the most prone to insomnia, and the rate of insomnia is high in elderly people. Working at stress, noise, nighttime, and family work imbalance was having impact. Factors indicating insomnia, effect of insomnia in other diseases as circadian rhythm disturbances, diabetes, and breast cancer were observed. The pathways involved, protein involved were analyzed, and it was observed as melatonin plays a key role in the inhibition of sleep induction. Treatments available are explored and enriched treatment with less side effects and good efficacy is needed.

Keywords: Age, Gender, Sleep, Sleep quality.

Indian Journal of Sleep Medicine (2020): 10.5005/jp-journals-10069-0053
\end{abstract}

Sleep is occupying one-third part of the time in one's life. A disturbance in such an important phase is a serious issue as it has an impact on daytime life. Insomnia is one of the sleep disorders. Insomnia is the most common problem which is ignored by ages. Half of the population is suffering from insomnia and it is expected to rise further in future due to our modern social lifestyle. For example, working systems, i.e., night shift works, professional stress, occupations dependent on electronic gadgets, round the clock working hours, and excess usage of social media. Insomnia is defined as the sleep disorder where one faces difficulty in having a healthy restful sleep. The lack of sleep results in multiple diseases. In order to reduce its risk, we need to focus on the study of insomnia, its classification, causative factors, diagnosis, treatment options, and pathways involved in analyzing the commonly ignored problem called insomnia. ${ }^{2}$

Insomnia is classified as acute insomnia and chronic insomnia, the latter one is commonly well known than the former. It is also classified into primary insomnia and secondary insomnia. The primary insomnia is sleeplessness, a psychological conditioning process that is not caused by medical, psychiatric, and environmental factors, whereas secondary insomnia is regarded as the medical psychiatric and environment causes this could be an underlying disorder. On the other hand of classification concerning the time, acute insomnia refers to the short-term which lasts for days or weeks caused by emotional or physical stress, chronic is long-lasting ongoing for months or more which has multivariable causes effecting this could be a side effect of other problem. As said above, chronic insomnia can be secondary insomnia. ${ }^{3}$ Symptoms of insomnia are difficulty in initiating or maintaining sleep, or early morning awakening. Symptoms of insomnia are also associated with hypertension cardiac risks, heart failure, and mortality. ${ }^{1}$

The overall mortality rate among men and women of mean age 50.2 and 47.4, respectively, was 21 men and 5\%, respectively. This study was adjusted to 14 years of follow-up for men and 10 years of follow-up for women. Individually, the men and women were grouped into four categories as normal sleep duration and
1,2Department of Biotechnology, National Institute of Technology, Warangal, Telangana, India

Corresponding Author: Perugu Shyam, Department of Biotechnology, National Institute of Technology, Warangal, Telangana, India, Phone: +91 9948561761, e-mail: shyamperugu@nitw.ac.in

How to cite this article: Shyam P, Mohammad MS. A Review Analysis of Insomnia: Age, Gender, Career, and Efficient Behavior Methods Perspectives. Indian J Sleep Med 2020;15(2):26-29.

Source of support: Nil

Conflict of interest: None

no insomnia, normal sleep duration with insomnia, short sleep duration with no insomnia, and short sleep duration with insomnia groups for men mortality rate was $9.1,12.6,31.0$, and $51.1 \%$, respectively, and for women $2.3,2.5,10.2$, and $2.5 \%$ was reported, respectively. Insomniac men whose sleep duration is less than a minimum of 6-hour sleep are prone to mortality risk. ${ }^{4}$ Insomnia risk rate in women is increased by 2.75 times by men as they reach menses and this continues the adult life due to the hormonal changes, pregnancy, medical conditions, stress, and complex home life. Chronic insomnia is reported in $80 \%$ of women who experience severe hot flashes. ${ }^{2}$

In the age-wise analysis, the estimated occurrence of insomnia in 4-year aged children is 36\%, 5-10 years aged is 20\%, $11-17$ years is $23.8 \%, 0.3-21 \%$ of infants carries on the symptoms to the schoolage, and $15-60 \%$ of school children carries on the symptoms to adolescent. ${ }^{5}$ It is reported that in children with Asperger syndrome or high-functioning autism, the presence of insomnia shows higher psychopathological symptoms. ${ }^{6}$ It is demonstrated in a recent analysis that $53 \%$ of adults lack 8-hour sleep, cognitive and somatic arousal are observed regularly in the adults prone to insomnia. Presleep arousal is increased in adults with sleep difficulties and these adults are detected to have an anxiety disorder. ${ }^{7}$ In elderly people over 60 years, insomnia is prevalent and $60 \%$ out of them are facing sleep difficulties. ${ }^{2}$ 
Insomnia is analyzed by career wise. It is seen frequent among shift workers, military persons, persons hearing harmful noises, and drivers. As of shift workers, for example, hospital emergency staff, nurses, security guards, software employees, customer care call center workers, and drivers. Due to nighttime working, sleepwake cycle is disturbed which associates with other distractions as a circadian cycle, body temperature, light-dark rhythm, and environment synchrony these all in term affect sleep and alertness. There is a new classification added by the International Classification of Sleep Disorder as "shift work sleep disorder" which is defined as having the symptoms of insomnia or excessive sleepiness due to the passing phase of shifts in work schedule. ${ }^{8}$ The military persons have reported post-traumatic stress as their work includes to be alert and active throughout their duty. ${ }^{9}$ Not only insomnia but also sadness, irritability, poor concentration, and brain traumatic injury are also prone to be affected. ${ }^{10}$ According to a study, the car crash or near-crash incidents are noticed by the insomniac drivers. ${ }^{11}$ The occupational noise can be major stress-inducing and increases the risk of physical and psychological factors as hearing loss, cardiovascular rhythm disturbance, mental health disturbance, and sleep disorders which eventually leads to insomnia. The main factors that influence the quality of sleep are body temperature, stress, age, work shifts, body mass index, lack of physical activity, smoking, and occupational noise stress. ${ }^{12}$ As core body temperature has a rhythm of declining toward environmental darkness and increases while morning promotes wakefulness, as well as skin temperature, is also affecting the same way. ${ }^{13}$

Insomnia and psychiatric distress are inter-relative. A 20-year cohort study states that psychiatric distress and chronic insomnia are linked with social and economic distractions. ${ }^{14}$ The effect of insomnia can adhere to many other diseases as breast cancer, insomnia-related diseases, diabetes, blood pressure, etc. Insomnia patients are more susceptible to cardiovascular diseases and mental health issues, these increase the mortality rate. ${ }^{15}$ It is observed that insomnia worsens the tumor of breast cancer. In African American breast cancer comparing to restful sleep, shorter sleep has more chances to triple-negative tumors. Deficient secretion of melatonin in darkness has its aggressive effects on sleep as well as breast cancer as it facilitates the increase of estrogenic circulation. ${ }^{16} \mathrm{~A}$ 20-year cohort study mentioned association between insomnia signs and diabetes 2 by a logistic regression method. ${ }^{17}$

Many methods of diagnosis are developed to measure the psychological aspects specifically as presleep arousal scale, the Presleep Cognitive Activity Questionnaire, the Metacognitions Questionnaire-Insomnia (MSQ-I), Dysfunctional and Attitudes about Sleep scale, and the Glasgow Sleep Effort Scale was designed to measure the attempt to control the process of falling asleep but all these are specific to use for clinical purpose. The most known instrument for the measurement of insomnia symptoms is the Insomnia Severity Index (ISI). PSQI Pittsburgh sleeps quality index is the most used diagnostic method. ${ }^{18}$ The diagnosis process is nothing but enquiring about the symptoms thorough sleep in the night as a cessation of breathing, snoring, gasping, repeated awakening, and excessive fatigue or irritability symptoms in the morning. The treatment underwent previously are also to be considered to diagnose. To accumulate information, a minimum of 2 weeks and more sleep log is needed. ${ }^{7}$

People facing insomnia often do not show up to a doctor for treatment, 70 out of 100 insomniacs neglect to consult a doctor. The treatment for insomnia is mostly performed by pharmacological, nonpharmacological, and acupuncture techniques. In nonpharmacological, the psychological treatment is well known, i.e., cognitive behavioral therapy in short called CBT. The CBT comprises five contents as cognitive understanding of sleep, seep hygiene, relaxation training, stimulus control, and sleep restrictions. The purpose and aim of this therapy are to prepare mind in perspective of sleep. This therapy is primarily opted for treating insomnia as it is less expensive, long last after therapy, and no adverse side effects. A new phase intervention of Internet CBT therapy known as SHUTi (Sleep Healthy Using The Internet) has a good impact on treating insomnia at low costs and feasible method. It comprises few stages to have a clear view on problem faced while sleeping and the appropriate treatment for the problem as shown in Figure 1, a step-by-step program. ${ }^{19}$

Coming to pharmacological treatment, eight medications were approved by the Food and Drug Administration (FDA) one of them is eszopiclone is approved to be used without restriction on duration. Five out of 100 people are noticed to take sedative medicines for a long time at night to help them sleep, but these sedatives have reported adverse effects as psychomotor issues. Elderly people are suggested to avoid sedative hypnotics as the risk overweighs the benefits. ${ }^{2}$ In pharmacological treatment, three main medications used are benzodiazepines, antidepressants, and antihistamines. In pharmacological treatment, there can be alternative medicine to sedatives which can be extracted from herbal plants as valerian from Valeriana officinalis, ginseng from Panax ginseng, kava kava from Piprer methystium, passionflower from Passiflora incarnata, hops from Humulus lupulus, and other aromatic plants like lavender, Melissa, chamomile, gelsemium, and jujuboside from Ziziphus jujuba. ${ }^{20}$ It is observed that sleep quality was accelerated in primary insomniacs with acupuncture treatment, but there are not many clinical or controlled trail studies performed on this treatment. ${ }^{3}$

Hormones like melatonin, serotonin, and prostaglandin-2 (PGD-2) are involved in insomnia. First, melatonin which regulates

Overview Step 1

- Defining insomnia

- Type of insomnia

- Setting up treatment overview, goals, effectiveness $\rightarrow$ Sleep behavior Step 2

- Explanation of poor sleep habits

- Sleep efficacy

- Instructing adjustments

Sleep behavior 2 Step 3

- Stimulus control

- Regular waking time in the morning

- No napping on bed when unsleepy

Sleep education Step 4

- Sleep hygiene guidelines

- Diet to follow

- Environment and exercise set up

Sleep thoughts Step 5

- Dealing misconception

- Dealing set backs

- Faulty thinkings

Problem prevention Step 6

- Prevention techniques

- Medication

- Repeat sleep behavior

Fig. 1: Step-by-step illustration of SHUTi program to treat insomnia through CBT-I 
circadian rhythms sleep-wake cycle is produced from the penial gland present in the center of the brain other than this melatonin has many impacts on biological functions as mood regulation, memory, learning, immunity, fertility, dreaming, and reproduction. ${ }^{21}$

This secretion of melatonin is increased as the evening turns to night and has its peak at 2-4 am facilitating to sleep in the darkness and reduces as the night gets diminishing facilitating to wakefulness. The secretion of melatonin is continuous all night to maintain the physiological level as it has a very short half-life, i.e., 30-40 minutes. The sleep-inducing properties of melatonin are due to the effect of melatonin receptors MT1 and MT2 located in the superchiasmatic nucleus in the hypothalamus as these regulate sleep and circadian rhythms. This hormone is quite effective in sleep regulation, anti-inflammation and with neuroprotective, precognitive properties, the most useful about melatonin is it is a naturally occurring hormone. ${ }^{22}$ L-Tryptophan is made into 5-hydroxytryptamine which is known to be serotonin and this is spread in central nervous system (CNS) in both vertebrates and invertebrates which acts as messenger regulator biochemically and local transmitter at synapses. ${ }^{23}$ This serotonin has a precursor 5-hydroxyl L-tryptophan which shows up in sleep disturbances and other circadian diseases. Prostaglandin-2 is a somnogen which has its main activity in the regulation of physiological sleep, PGD-2 is involved in contribution to insomnia. Androstenol a neurosteroid has GABAA receptor modulator activity, it is observed consistently in all animal models. ${ }^{24}$

Drug target discovery for the diseases through metabolic pathway analysis is the novel approach. The key component involved in a specific pathway to a disease condition is called drug target. The drug is designed as the target key molecules are enhanced or inhibited which have their influence on the whole metabolic pathway. During the possible pathway analysis, the links between pathways may entangle in metabolic networks creating a problem of combinatorial explosion. So, there is an eager need to develop new methods to have an efficient, potential drug target discovery. Presently, sources for metabolic pathway information are

- IPA: International Pharmaceutical Abstracts

- KEGG: Kyoto Encyclopedia of Genes and Genomes

- HMDB: Human Metabolome Database

where we can identify and explore drug targets, metabolites, and their relation. In a recent study, Wang et al. have identified six unique pathways to the insomnia suffering animals through MetPA. Potential drug targets were also identified from pathways related to

- Ascorbate and aldarate metabolism.

- Tryptophan metabolism.

- Starch and sucrose metabolism

- Amino sugar and nucleotide sugar metabolism.

- Pantothenate and CoA: Coenzyme-A biosynthesis.

- Pentose and glucoronate interconversions.

Sixteen different targets were identified from the above pathways involving in stages of insomnia. It is observed that ascorbate and aldarate metabolism has a high score. These target pathways are involved in insomnia and can develop insomnia. In the circadian entrainment pathway, the involvement of melatonin is observed. As in Figure 2, the pathway, melatonin is released from the pineal gland at dusk dawn condition and 2 receptors as MT1 and $\mathrm{MT} 2$ are observed which are coupled with $\mathrm{G}$-protein reactions with guanine nucleotide-binding protein $\mathrm{G}(\mathrm{i}), \mathrm{G}$ (q) subunit alpha. These inhibits enzyme adenylate cyclase 1 (AC) [EC: 4.6.1.1]. These are linked further to protein kinase cascades (PKC) the release of $\mathrm{k}+$ ions is shown to inhibit the induction of sleep in the pathway.

At last, it can be concluded that why insomnia is to be focused as a serious problem because it is itself a prevalent sleep disorder and upon that it has the lead to many other serious health issues as cancer, diabetes, blood pressure, and circadian rhythm problems.

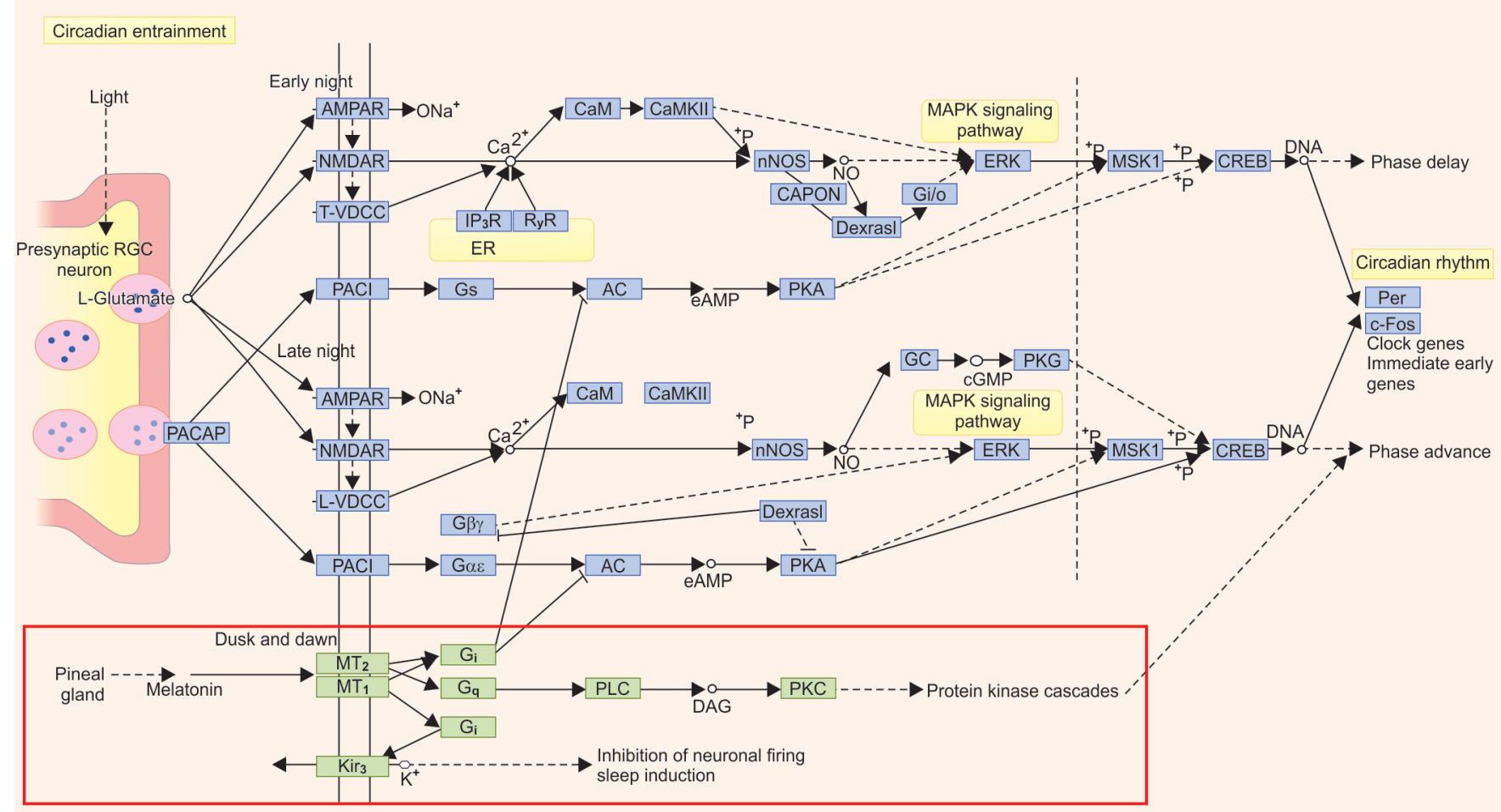

Fig. 2: Circadian entrainment pathway collected from KEGG: Kyoto Encyclopedia of Genes and Genomes tool. Action of melatonin in pathway is highlighted 
The ratio of effect of insomnia is directly proportional to the worsening of other diseases. It can be seen that women belonging to the postmenopause state, and elderly people are the most affected persons out of society. Insomnia and its prevalence are dependent on the nature of work people choose for living. It is observed that the nature of work which includes night shift works, hearing heavy noises, stress-inducing, high exposure to radiations, and driving at night times are much ready to face insomnia. So, choosing a career have a role in sleep disorder motivation. The treatment for insomnia is under known to many people even at this time as only a few people make sure the problem of sleep and consult a doctor. To treat this, there are most preferred two means as pharmacological and psychological. The psychological treatment of insomnia is performed by giving cognitive behavioral therapy which is performed by psychiatrists and neurologists but not possible clinically. In recent times, there is much growth in online therapy called SHUTi which is providing Internet-based CBT treatment. This is low of cost, feasible with timing, and the most importantly anywhere accessible course which is not well known. These psychological treatment shows mean results compared to pharmacological treatment. Clinically, pharmacological treatment is possible but the drawback observed is it has side effects that are balancing out the benefits of its efficacy. So, there is an extensive thrive need to fill the gap for the efficient treatment of insomnia with reducing side effects. In recent observations, many herbal medicinal plants are producing the components involving in the metabolic pathway of insomnia. These medicinal plant-based extractions can be concentrated to achieve an efficient drug to treat insomnia with no or low side effects for the next generation. Furthermore, these plant-based drugs are to be clinically tested on animal models and approved by the FDA to have their complete achievement.

\section{References}

1. Lisan Q, Tafflet M, Thomas F, et al. Body silhouette trajectories over the lifespan and insomnia symptoms: the paris prospective study 3. Sci Rep 2019;9(1):15-81. DOI: 10.1038/s41598-018-38145-7.

2. Hamblin JE. Insomnia: an ignored health problem. Primary Care Clinics in Office Practice 2007;34(3):659-674. DOI: 10.1016/ j.pop.2007.05.009.

3. Attele AS, Xie JT, Yuan CS. Treatment of insomnia: an alternative approach. Altern Med Rev 2000;5(3):249-259.

4. Vgontzas AN, Liao D, Pejovic S, et al. , Insomnia with short sleep duration and mortality: the Penn state cohort. Sleep 2010;33(9): 1159-1164. DOI: 10.1093/sleep/33.9.1159.

5. Combs D, Goodwin JL, Quan SF, et al. Insomnia, health-related quality of life and health outcomes in children: a seven-year longitudinal cohort. Sci Rep 2016;6(1):27921. DOI: 10.1038/srep27921.

6. Allik H, Larsson JO, Smedje H. Insomnia in school-age children with asperger syndrome or high-functioning autism. BMC Psychiatry 2006;6(1):18. DOI: 10.1186/1471-244X-6-18.

7. Blake M, Schwartz O, Waloszek JM. The SENSE study: treatment mechanisms of a cognitive behavioral and mindfulness-based group sleep improvement intervention for at-risk adolescents. Sleep 2017;40(6):zsx061. DOI: 10.1093/sleep/zsx061.

8. Garbarino S, Repice AM, Traversa F, et al. Sleep disorders in shift worker. Sleep 2002;25(6):642.
9. Lee SW, Lee SM, Shin SY, et al. The effect of morningness, eveningness type of shift working nurses on sleep quality, depressive symptom, and occupational stress. Sleep 2017;(1):40. DOI: 10.1148/ radiol.2017162148.

10. Tegeler CL, Gerdes L, Shaltout HA, et al. Successful use of closed-loop allostatic neurotechnology for post-traumatic stress symptoms in military personnel: self-reported and autonomic improvements. Mili Med Res 2017;4(1):38. DOI: 10.1186/s40779-017-0147-0.

11. Shu-Yuan Liu, et al. The impact of sleep disorders on driving safetyfindings from the Second strategic highway research program naturalistic driving study. Sleep J 2018;41(4):zsy023.

12. Test T, Canfi A, Eyal A, et al. The influence of hearing impairment on sleep quality among workers Exposed to harmful noise. Sleep 2011;34(1):25-30. DOI: 10.1093/sleep/34.1.25.

13. Benjamins JS, Migliorati F, Dekker K, et al. Insomnia heterogeneity: characteristics to consider for data-driven multivariate subtyping. Sleep Med Rev 2017;36:71-81. DOI: 10.1016/j.smrv.2016.10.005.

14. Green MJ, Espie CA, Benzeval M. Social class and gender patterning of insomnia symptoms and psychiatric distress: A 20-year prospective cohort study. BMC Psychiatry 2014;14(1):152. DOI: 10.1186/1471-244X14-152.

15. van der Zweerde T, Lancee J, Slottje P, et al. Cost-effectiveness of i-sleep, a guided online CBT intervention, for patients with insomnia in general practice: protocol of a pragmatic randomized controlled trial. BMC Psychiatry 2016;16(1):85. DOI: 10.1186/s12888-016-0783-z.

16. Soucise $A$, Vaughn $C$, Thompson $C L$, et al. Sleep quality, duration, and breast cancer aggressiveness. Breast Cancer Res Treat 2017; 164(1):169-178. DOI: 10.1007/s10549-017-4245-1.

17. Green MJ, Espie CA, Popham F, et al. Insomnia symptoms as a cause of type 2 diabetes incidence: a 20 year cohort study. BMC Psychiatry 2017;17(94):6-8. DOI: 10.1186/s12888-017-1268-4.

18. Crönlein $T$, Langguth $B$, Popp $R$, et al. Regensburg insomnia scale (RIS): a new short rating scale for the assessment of psychological symptoms and sleep in insomnia; study design: development and validation of a new short self-rating scale in a sample of 218 patients suffering from insomnia and 94 healthy controls. Health Qual Life Outcomes 2013;11(1):65. DOI: 10.1186/1477-7525-11-65.

19. Brooks AT, Tuason RT, Chakravorty $S$, et al. Online cognitive behavioral therapy for insomnia (CBT-I) for the treatment of insomnia among individuals with alcohol use disorder: study protocol for a randomized controlled trial. Pilot Feasibility Stud 2018;4(1):183. DOI: 10.1186/s40814-018-0376-3.

20. Wheatley $D$. Medicinal plants for insomnia: a review of their pharmacology, efficacy and tolerability. J Psychopharma 2005;19(4):414-421. DOI: 10.1177/0269881105053309.

21. Wang X, Yang B, Zhang A, et al. Potential drug targets on insomnia and intervention effects of jujuboside a through metabolic pathway analysis as revealed by UPLC/ESI-SYNAPT-HDMS coupled with pattern recognition approach. J Proteom 2012;75(4):1411-1427. DOI: 10.1016/ j.jprot.2011.11.011.

22. Baandrup L, Fagerlund B, Jennum $P$, et al. Prolonged-release melatonin versus placebo for benzodiazepine discontinuation in patients with schizophrenia: a randomized clinical trial - the SMART trial protocol. BMC Psychiatry 2011;11(1):160. DOI: 10.1186/1471-244X11-160.

23. Lee PT, Lin HW, Chang $\mathrm{YH}$, et al. Serotonin-mushroom body circuit modulating the formation of anaesthesia-resistant memory in drosophila. Proc Natl Acad Sci U S A 2011;108(33):13794-13799. DOI: 10.1073/pnas.1019483108.

24. Ahmad AS, Ottallah H, Maciel CB, et al. Role of the L-PGDS-PGD2-DP1 receptor axis in sleep regulation and neurologic outcomes. Sleep 2019;42(6):zsz073. DOI: 10.1093/sleep/zsz073. 